\title{
Contribution à l'étude des mouvements solides provoqués par la houle sur un fond horizontal
}

\section{Contribution to the study of sediment transport on a horizontal bed due to wave action}

\author{
G. VINCENT \\ INGÉNIEUR \\ ET \\ F. RUELLAN \\ ASSISTANT
}

PAR

SOGREAH (LAGORATOIRE DAUPHINOIS D'HYDRAULIQUE)

En vue de préciser le phénomène des monvements solides en mer, au large de la zone de déferlement, les auteurs ont étudié l'action de la houle sur un fond horizontal. Ils donnent un certain nombre de résultats nouveanx concernant :

1. L'état de turbulence au voisinage du fond: stabilité de la conche limite laminaire oscillatoire.

2. Le début de mouvement des matériaux sous l'influence de la houle seule.

3. Le courant d'entrainement provoqué par la honle au voisinage immédiat du fond.

1. Le transport des materiaux sous linfluence de la houle seule.

5. L'action indirecte de la houle sur les fonds. Les conclusions principales sont les suivantes:

(1) Les résultats donnés par Huon Li concernant l'apparition de la turbulence dans la conche limite oscillatoire äonnent trop d'importance au laminaire. Vo (vitesse orbitale maximum) et $\varepsilon$ (rugosité) sont les facteurs principaux caractérisant la transition.

La houle expérimentale n'est généralement pas (ou peu) turbulente dans la masse, elle est, par contre, le plus sauvent turbulente au voisinage immédiat du fond.

(2) L'étude des conditions de début de mouvement des grains de matériau constituant le fond révèle que l'action de la houle peut être notable, même par des fonds de plusieurs dizaines de mètres. Une houle d'amplitude totale $6 \mathrm{~m}$. longneur d'onde totale $120 \mathrm{~m}$ serait capable de mettre en mouvement un grain de sable de $0,3 \mathrm{~mm}$ par $60 \mathrm{~m}$ de fond.

(3) L'étude expérimentale, comme la théorie en fluide visqueux, fait appuraitre l'existence, an voisinage immédiat du fond, d'un courant d'entrainement des particules liquides portant dans le sens de propagation de la houle.

(4) Ce courant d'entraînement (qui, en canal d'essais, s'accompagne d'un courant de transport dans la masse fluide dont la distribution
With a view to explaining the phenomena of sediment transport in the open sea, outside the wave breaking area, the authors carried ont a laboratory investigation of wave action on $\alpha$ horizontal bed. They put forward a number of new results regarding:

1 The state of turbulence near the bed and the stability of the oscillatory laminar boundary layer.

2 The setting in molion of malerials under the influence of wave alone.

3 The entrainment current caused by wave action close to the bed.

' The transport of material under wave action only.

5 The indirect action of wave on the bed.

The main conclusions reached are as follows :

(1) The results given $b_{y}$ Huon Li regarding the onsel of turbulence within the oscillatory boundary layer overestimate the range of laminat conditions. Vo (maximum orbital velocity) and \& (roughness) are the principle factors governing the transition.

Test waves are either generally laminar, or are only slightly turbulent within the body of liquid, but they are, however, more often turbulent in the immediate neighbourhood of the bed.

(2) The investigation of conditions for the onset of grain movement of the bed material shows that the action of wave can be appreciable, even at depths of several tens of metres. A wave of 6 metres amplitude, with a total lenglh of 120 metres, would be capable of putting a $0.3 \mathrm{~mm}$ sand grain into motion at a depth of 60 metres.

(3) The experimental investigation, as well as lhe viscous fluid theory, shows the existence, close to the bed, of an entrainment current of liquid particles which always worls in the direction of wave propagation.

(1) In test flumes, this entrainment current 
verticale varie selon les caractéristiques relatives au fluide) comme la prépondérance, an voisinage da fond notamment, de la vitesse des particules liquides dans le sens de sa propagation, donnent lieu généralement sur un fond horizontal $\dot{a}$ un transport solide effectif dans le sens de propagation de la houle.

(5) Du fait de l'existence du courant de transm port de masse et de la mise en suspension des matériaux au-dessus du fond, un certain transport solide peat exister vers le large. Ces résultats permettent d'expliquer qu'en houle allongée et régulière, les matérianx sont de préférence charriés dans le sens de la houle et biennent engraisser les rivages tandis qu'en tempète une turbulence résultante importante donne de la suspension et favorise l'érosion de ces rivages.

(6) Sur un fond en pente, le transport vers la côte est contrebalancé par l'effet de la gravits. les courants provoques par les vents du large et les courants de densité prenant naissance dans la zone de deferlement, si bien que fina lement les matériaux érodés du continent se perdent, en partie, lentement vers le large. forms part of a mass transport within the li quid, the vertical distribution of which varies with the characteristics of the fluid motion. On a horizontal bed, it generally gives rise to an effective sediment transport, in the direction of wave propagation, as the preponderant part of the liquid velocity component, near the bed, is in this direction.

(5) Ouing to the existence of the mass transport current and the onset of suspension of material above the bed, some sediment transport can exist out to sea. These results give an explanation of why, under the action of long and regular wave, material tends to be carried in the direction of the waves and build ap on the beach whereas, under storm conditions, $a$ strong resultant turbulence produces suspension and favours erosion of the beach.

(6) On a sloping bed, transport towards the shore is counterbalanced by the effect of grivity, currents caused by winds from seaward and density currents set up in the wave breaking area so that finally material eroded from land surfaces are, in part, gradually carried away towards the open sea.

\section{GÉNÉRALITÉS}

Nous aborderons ici le problème du transport solide par la houle sur le fond de la mer, e'està-dire au large de la zone de déferlement, soit hors de la zone où se manifeste principalement ce que nous avons coutume d'appeler le transport littoral.

\section{a) Action de la houle sur le fond de la mer. - Généralités.}

De la zone de déferlement jusqu'à des profondeurs assez grandes, l'action de la houle sur les fonds se manifeste avec une ampleur qui dépend, outre de la nature "in situ» des fonds, de la profondeur d'ean et des caractéristiques des vagues. Généralement, sur ces profondeurs, les matériaux des fonds sont mis en mouvement sans qu'il y ait nécessairement des déplacements importants de particules solides et l'on peut dire que l'intensité de ces déplacements est d'autant plus faible que l'on s'écarte de la zone de déferlement. Cette décroissance doit être en général très rapide, car la turbulence, au voisinage du fond en particulier, joue un rôle notable sur le transport solide et lorsqu'on s'éloigne du déferlement vers le large, celle-ci doit en réalité diminuer rapidement au sein des couches inférieures du fluide. Ceci explique que, généralement, la suspension s'atténue rapidement vers le large, et que, corrélativement d'ailleurs, les matériaux qui se déposent deviennent de plus en plus fins.

Néanmoins, il ne faut pas perdre de vue qu'au large de la zone de déferlement, la houle n'est pas le seul facteur naturel capable de transporter des matériaux. Les particules solides mises en mouvement par la houle pourront être reprises par un courant marin quelconque, même très faible; celui-ci agira en effet sur des grains de matériau déjà arrachés au fond.

\section{b) Technique expérimentale.}

La plupart des auteurs qui ont étudié expérimentalement soit l'état de turbulence, soit les mouvements solides dus à l'action de la houle se propageant en eau peu profonde, sur un fond horizontal : Huon Li, Manohar, Bagnold ,etc., ont opéré en faisant osciller le fond dans une masse d'eau tranquille.

Si cette méthode permet d'obtenir, au voisinage du fond, des mouvements dont l'ampleur est du même ordre de grandeur que ceux de la réalité, elle ne reflète néanmoins qu'incomplètement l'influence que peuvent avoir, sur une couche de sédiments au repos, différents phénomènes auxquels donne naissance une houle progressive (courant d'entraînement, accélérations des particules liquides, fluctuations de pression au voisinage immédiat du fond, développement de la turbulence, etc.).

Aussi, et bien que cette méthode permette d'obtenir des résultats moins directement applicables à la réalité (en raison de la gamme moins étendue des vitesses considérées), nous avons préféré conduire nos études expérimentales dans un canal à houle classique dans lequel les maté- 
riaux constituant le fond étaient soumis à l'action effective d'une houle progressive.

\section{Notations}

Notations utilisées dans la suite du texte:

$2 a$ : amplitude totale de la houle à la surface libre (distance verticale de creux à crête);

$2 \mathbf{a}_{d}$ : amplitude totale de la houle correspondant au début du mouvement d'un grain de matériau;

$d$ : diamètre d'un grain de matériau de la couche constituant le fond; $d$ est choisi de telle manière que $50 \%$ des grains ont un diamètre inférieur;

$d_{1}$ : excursion totale d'une particule liquide au voisinage immédiat du fond:

$d_{1}=2 a /(\operatorname{sh} 2 \pi h / L)$ (valeur théorique, premier ordre, fluide parfait);

$5 \quad$ : épaisseur de la couche laminaire oscillatoire $\left(\delta=6,5 d_{0}\right)$;

$\varepsilon \quad$ : dimension caractéristique d'un grain du matériau pulvérulent constituant le fond. (En fait, pour un matériau de granulométrie uniforme, $\varepsilon \equiv d)$; $\hat{g} \quad$ : champ de pesanteur apparent

$$
\hat{g}=g\left(\rho_{s}-\rho_{f}\right) / \rho_{f} \text { : }
$$

$h$ : profondeur d'eau au-dessus dn fond;

L. : longueur d'onde de la houle (distance horizontale entre deux crètes successives);

$\checkmark \quad$ : viscosité cinématique de l'eau;

$\rho_{3}$ : masse spécifique du grain;

\% : masse spécifique du fluide;

$\mathrm{T}$ période de la houle $(\omega=2 \pi / \mathrm{T})$;

ii : intensité maximum du courant de masse au voisinage du fond en régime laminaire (valeur moyenne);

$u(z)$ : vitesse de l'écoulement dans la couche limite laminaire oscillante à la cote $z$ audessus du fond;

$u_{z} \quad$ : intensité du courant de masse à la cote $z$ au-dessus du fond;

$\mathrm{V}$ : vitesse d'une particule liquide à la distance $\delta \mathrm{du}$ fond;

$\mathrm{V}_{\mathrm{n}} \quad$ : valeur maximum de $\mathrm{V}$; elle est égale à la vitessse orbitale théorique maximum au voisinage immédiat du fond;

IV : vitesse de chute en eau calme d'un grain de matériau de diamètre $d$.

\section{I. - ETAT DE TURBULENCE AU VOISINAGE DU FOND STABILITE DE LA COUCHE LIMITE LAMINAIRE OSCILLATOIRE}

\section{a) Epaisseur de la couche limite laminaire.}

Le système des forces hydrodynamiques agissant sur un grain de matériau de la couche constituant le fond dépendra principalement des conditions de l'écoulement au voisinage immédiat du fond, e'est-à-dire, en fait, de la nature de l'écoulement autour dụ grain.

Or, pour un fluide visqueux, du fait de l'écoulement général - même si celui-ci est oscillatoire - une couche limite à fort gradient de vitesse - elle-même oscillatoire - se développera sur le fond.

De manière générale, l'apparition de la turbulence au sein de cette couche limite oscillatoire peut être considérée comme étant un facteur important $\mathrm{du}$ transport solide au voisinage du fond. influant tout spécialement sur le début de mourement des grains.

On conçoit en effet aisément que la nature de l'écoulement autour du grain dépende du rapport $\delta / \varepsilon$, e'est-à-dire du degré d'immersion du grain dans la couche limite laminaire oscillatoire.

\section{b) Stabilité de la couche limite : Etudes de Huon Li.}

La stabilité de la couche limite oscillatoire a fait récemment aux U.S.A. l'objet d'une étude expérimentale très intéressante de Huon Li, complétée par Manohar.

L'étude expérimentale de ce phénomène en houle progressive se heurte évidemment à de grandes difficultés si l'on désire reproduire, même schématiquement sur un modèle, les conditions aux limites résultant de l'action de la houle sur un fond naturel.

Pour son étude expérimentale, Huon Li a opéré comme l'avait fait Bagnold, en faisant osciller, dans un plan horizontal, le fond lui-même au sein d'une masse liquide au repos.

Cette manière d'opérer fait notamment abstraction des accélérations périodiques auxquelles sont soumises les particules fluides (*), ainsi que des variations de pression au voisinage du fond; les unes et les autres doivent avoir une certaine

(c) Voir notamment R. Miche. 
tendance à faire en quelque sorte "éclater» la couche limite laminaire, c'est-à-dire à accélérer l'apparition de la turbulence au sein de celle-ci. II faut, en conséquence, s'attendre à ce que les résultats de Huon $\mathrm{Li}$ donnent relativement trop d'importance au laminaire par rapport à ce qu'il doit être réellement lorsque la couche limite oscillatoire est formée par une houle progressant effectivement sur un fond au repos.

Les résultats obtenus au cours de notre étude expérimentale sont en accord avec cette remarque. Ils confirment en outre que la transition apparait d'autant plus rapidement que la dimension caractéristique du grain, l'excursion d'une particule liquide au voisinage immédiat du fond et la fréquence du mouvement oscillatoire sont importants (c'est-à-dire, en fait, la vitesse d'une particule liquide au voisinage immédiat du fond).

\section{c) Définition d'un critère de "transition $"$}

Lorsque la couche limite oscillatoire est laminaire, on constate qu'une fine particule de colorant abandonnée sur le fond donne naissance à un nuage coloré de faible épaisseur (en un point donné, cette épaisseur varie légèrement au rythme de la houle) dont la face supérieure apparaît parfaitement lisse ('une des extrémités du nuage progresse dans le sens de propagation de la houle).

L'épaisseur e moyenne du nuage coloré croît légèrement lorsque la vitesse de la particule liquide an voisinage du fond croit (fig. 1).

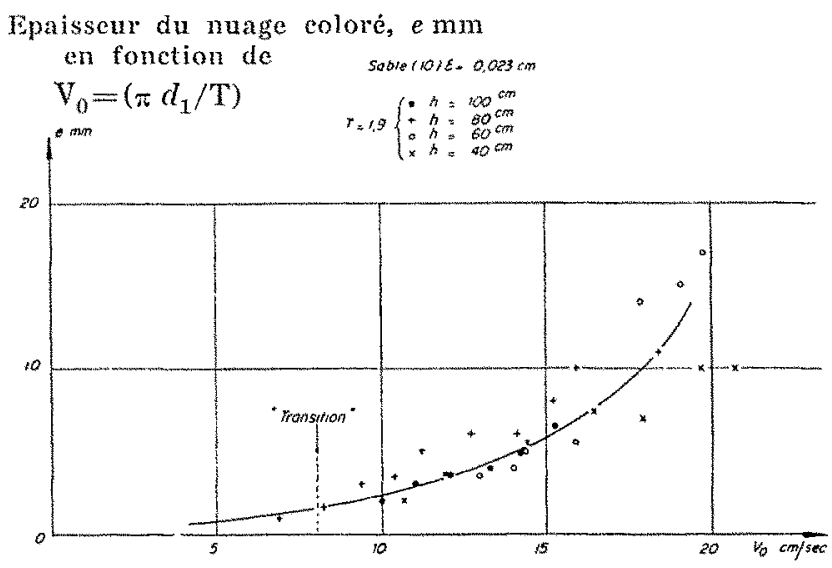

Fia. 1 ,

Pour une certaine vitesse d'oscillation, de légères discontinuités apparaissent sur la face supérieure du nuage. Ces discontinuités se forment alors que la pression sur le fond est au voisinage de son minimum; du fait de l'écoulement local momentané, elles sont infléchies dans un sens opposé à celui de la houle. La vitesse croissant encore, elles se développent en « petites flam- mes $\gg$ individuelles d'ampleur progressivement croissante.

Nous avons considéré que l'apparition de ces «flammes » caractérisait la «transition».

Leur apparition, toutes choses égales par ailleurs, se manifeste pour une vitesse d'oscillation d'autant plus faible que la « rugosité » du fond est elle-mème grande.

On peut déceler, au contact immédiat du fond, un film laminaire dont l'épaisseur, relativement très faible, décroît lorsque la turbulence locale croît.

La couche limite étant turbulente, si l'on abandonne à partir de la surface libre un grain coloré de densité voisine de celle du fluide, ce grain descend lentement en tracant dans la masse un filet coloré très bien individualisé jusqu'à une distance du fond d'autant plus réduite que la * turbulence » est elle-même faible.

Ceci tend à montrer qu'en fait, en houle régulière, la turbulence produite par le fond se propage assez mal dans la masse fluide.

\section{d) Résultats de Huon Li.}

Selon les expériences de Berkeley, le fond lisse - au sens hydraulique du mot - existerait si : $\delta / \varepsilon>30$; par contre, le fond serait « rugueux 》 si : $\hat{o} / \varepsilon<18,5$.

Huon Li caractérise l'épaisseur de la couche laminaire oscillatoire par l'expression :

$$
\delta=6,5(v / \omega)^{1 / \alpha} \text {, soil } \delta=2,6 \cdot \sqrt{v T}
$$

Cette valeur est sans doute une valeur par excès; néanmoins, dans un but de comparaison, nous avons retenu ici la même définition.

Selon Huon Li, pour un fond « lisse», la couche limite oscillatoire devient turbulente pour un nombre de Reynolds local, tel que:

$$
d_{1}(\omega / v)^{1 / 2}=800
$$

ceci même, semble-t-il, pour des valeurs de $v / \omega$ relativement faibles.

Pour un fond "rugueux », le nombre de Reynolds de transition $\omega d_{1} s / \nu$ est une constante pour chaque rugosité, c'est-à-dire en fait pour chaque dimension caractéristique des grains du matériau constituant le fond.

\section{c) Résultats Sogreah.}

Nous avons porté (fig. 2) les valeurs $d_{1}=f(T)$ pour lesquelles, selon le critère défini précédemment, la turbulence apparaî dans la couche limite oscillatoire. En pointillés, les valeurs obtenues à Berkeley pour le fond « lisse » et divers matériaux, en traits continus les valeurs obte- 
nues au cours de nos expériences en houle progressive.

On constate que, pour deux matériaux de $d$ (ou $\varepsilon$ ) voisins et pour une période donnée du mouvement oscillatoire, la turbulence apparaît pour une valeur plus faible de l'amplitude totale d'oscillation lorsque le mouvement relatif an voisinage du fond est le résultat de l'action d'une

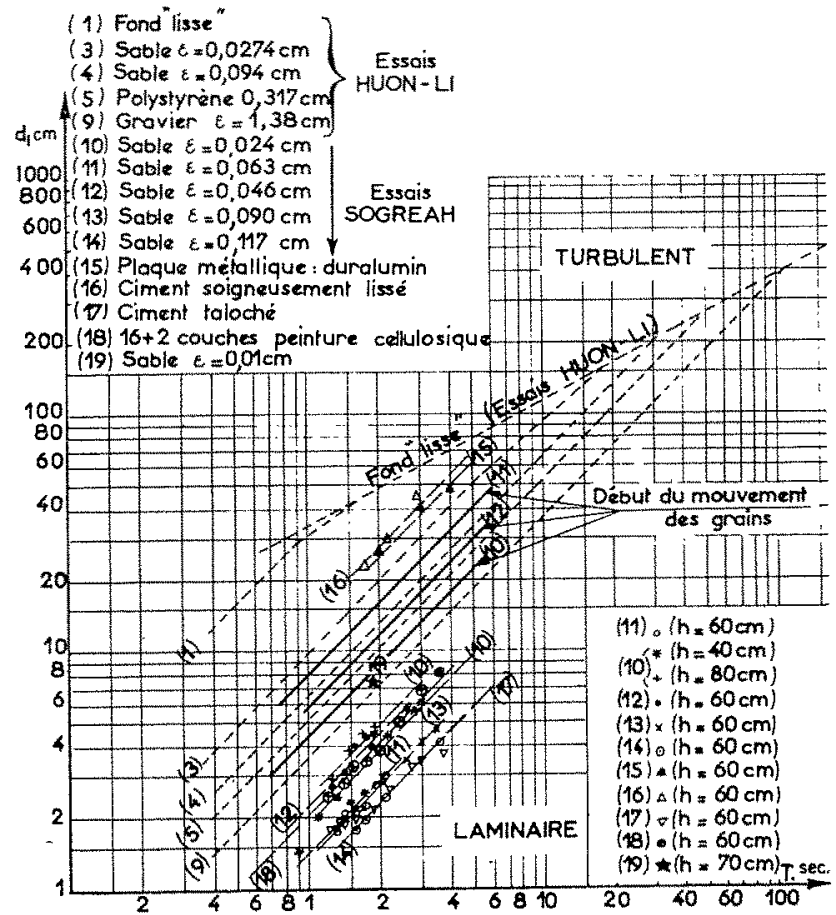

Fig. 2. - Apparition de la turbulenee dans la conche limite laminaire oseillatoire en houle progressive.

houle. (Si nous considérons deux valeurs voisines de $\varepsilon$ par exemple, les matériaux (3) et (10) ou (4) et (13), pour une même valeur de $T$ la transition apparaît, selon les résultats de Huon Li, pour une valeur de $d_{1}$ environ cinc fois supérieure à celle que nous avons nous-mêmes notée.)

Cette différence dans les résultats expérimentaux est, semble-t-il, assez générale, elle se retrouve quelle que soit en valeur absolue la « rugosité » du fond (cette rugosilé étant constituce par les grains de matériau de dimension caractéristique $\varepsilon$, soit en fait $d$ ).

II n'est pas possible d'affirmer que cette différence se retrouve pour un fond « lisse » ćtant donné les difficultés qu'il y a à caractériser l'état d'une telle surface. Bien que nous ayons considéré des fonds relativement très lisses au sens donné par Huon $\mathrm{Li}(\delta / \varepsilon>30)$ - plaque métallique lisse au sens physique, ainsi que ciment soigneusement lissé - du point de vue de la transition ceux-ci se comportaient, semble-t-il, en fond rugueux en ce sens que la transition se pro- duisait pour une valeur de nombre de Reynolds encore faiblement dépendante de l'état de surface $\left(^{*}\right)$.

Il est certes possible qu'une légère différence dans le choix des criteres de transition retenus tant à Berkeley" qu'à Grenoble puisse expliquer une faible part des divergences constatées. Il est plus vraisemblable néanmoins que celles-ci rèsultent des différences expérimentales elles-mêmes. $\mathrm{Si}$, en effet, à titre de curiosité, on porte sur le graphique $d_{1}=f(\mathrm{~T})$ caractérisant la transition, pour les trois sables considérés, (10), (11), (12), des valeurs de $d_{1}$ pour lesquelles se manifeste le début de mouvement des grains constituant le fond, on constate que ce début de mouvement des grains se manifeste pour une valeur de $d_{1}$ ellemême inférieure à celle par laquelle $\mathrm{M}$. Huon $\mathrm{Li}$ caractérise la transition. Dans ces cas pourtant, le début de mouvement se manifestait alors que la couche limite semblait manifestement être turbulente.

En résumé, les valeurs de Berkeley semblent donner trop d'importance au laminaire.

INTEUENCE DE LA POROSITE DU FOND SUR L'APPARITION DE LA TURBULENCE

Les essais avec les sables 10,11 et 13 ont élé répétés en couche dite épaisse (couche de matériau de $5 \mathrm{~cm}$ d'épaisseur) et en couche dite mince (couche de matériau ayant l'épaisseur de quelques grains, les grains de la conche inférieure étant collés sur une surface lisse imperméable).

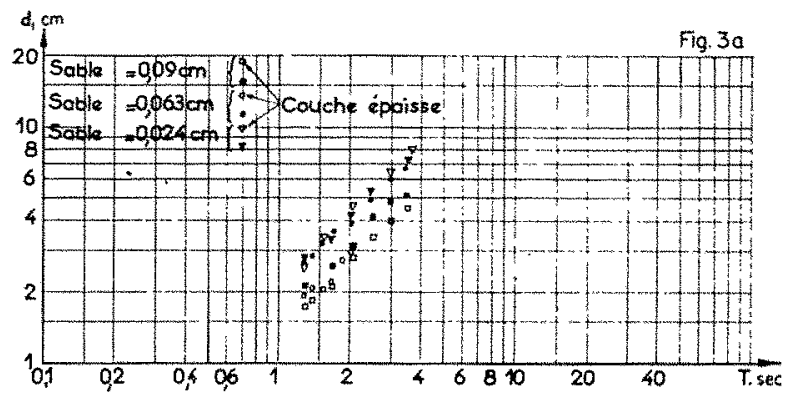

Frg. $3 a$. - Influence de la porosite du fond sur lapparition de la turbulence.

Les résultats sont portés sur le graphique fig. $3 a$. Il apparait que la porosité du fond facilite dans une certaine mesure lapparition de la turbu-

(*) Il est vraisemblable que si nous avions considere comme l'a fait Huon lii un fond plus lisse au sens physique, par exemple une platue de verre poli, nous aurions atteint le domaine du fond hydrauliquement lisse. Les résultats obtenus avec la plaque métallique et le ciment lissé conduisent à penser que le nombre de Reynolds $\omega^{1 / 2} d_{1} v^{-1 / 3}$, caractérisant la transition pour un fond hydrauliquement lisse, n'aurait pas été inférieur à 800 . 


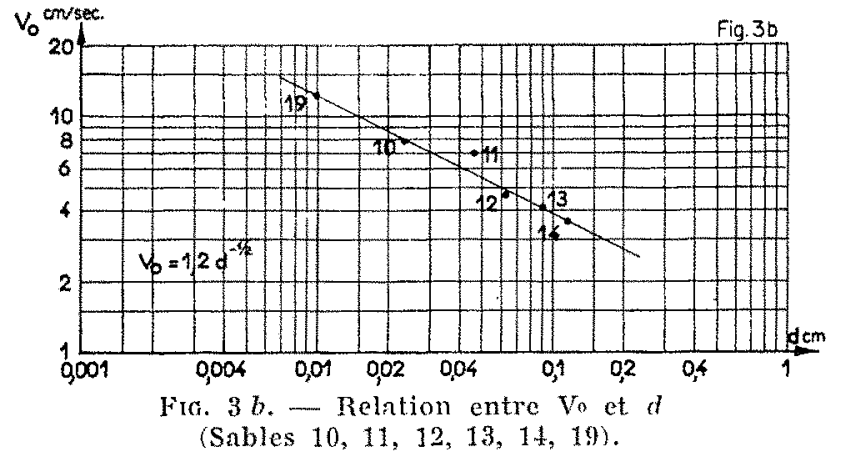

lence; cette influence devient tris faible pour les sables de diamètre inférieur à $0,024 \mathrm{~cm}$.

\section{INFLUENCE DE DIAMETRE DU GRAIN}

Mise à part l'épaisseur de la couche de matériau, le diamètre $d$ du grain et $V_{0}$ sont les paramètres principaux caractérisant la transition, Entre ces deux paramètres, on peut, selon nos résultats, définir une relation de la forme $\mathrm{V}_{0}=1,2 d^{-1 / 2}$ (fig. $3 b$ ).

\section{II. - DEMARRAGE DES GRAINS}

Si l'on considère l'évolution du mouvement au voisinage immédiat du fond à partir de la naissance de la couche limite laminaire jusqu'au moment où un transport solide caractérisé se manifeste, on constate en général les phénomènes suivants :

1. Développement de la couche limite laminaire oscillatoire;

2. Apparition de la turbulence dans la couche limite;

3. Début de mouvement des premiers grains de matériaux $\left({ }^{*}\right)$ (les premiers grains transportés sont souvent les plus gros de ceux constituant la couche de matériau du fond);

4. Mouvement généralisé des grains;

5. Apparition des rides et régularisation de celles-ci;

6. Transport solide caractérisé par charriage et saltation dans le sens de propagation de la houle;

7. Lente progression de la turbulence vers la masse du fluide, du fond vers la surface;

8. Premières manifestations d'un transport en suspension dans la partie inférieure de la masse liquide dans le sens inverse du sens de propagation de la houle;

9. Allongement et disparition progressifs des rides.

(*) Il peut néanmoins très bien se produire que le début de mouvement des grains précède l'apparition de la turbulence si le matériau est particulièrement fin. Selon nos résultats expérimentaux, cette éventualité parait se présenter, pour des périodes de l'ordre de 2 secondes, lorsque $\varepsilon \leqslant 0,01 \mathrm{~cm}$. Cette remarque découle d'ailleurs directement des résultats présentés fig. 3 et fig. 5 ; en effet, pour une période donnée de la houle $d_{1}$ décroît quand $d$ croit (transition) tandis que $d_{1}$ croit quand $d$ croît (démarrage des grains).

\section{a) Résultats antérieurs.}

R. A. Bagnold est, à notre connaissance, le premier qui ait abordé ce problème en étudiant le démarrage des grains de matériau constituant une couche d'épaisseur uniforme reposant sur une surface cylindrique oscillant dans une masse d'eau au repos.

En 1954, des essais en houle progressive onl été effectués à l'Institut de Mécanique des Fluides de Lille par MM. Martinot-Lagarde et Fauquet.

Arthur T. Ippen et Peter S. Eagleson, puis Madhar Manohar aux U.S.A. ont également abordé ce problème ces toutes dernières années. Plus récemment encore, M. Larras a étudié les effets de la houle et du clapotis sur les fonds de sable. A la suite de ces essais, M. Larras a donné une relation entre $V_{0}$, T et $M$, caractérisant le début de mouvement.

\section{b) Critère de début de mouvement.}

L'expérience montre que lorsque l'on parle de début de mouvement d'un matériau constituant un fond mobile, il est essentiel de bien préciser le critère de démarrage; a fortiori, si l'on veut comparer entre eux des résultats expérimentaux obtenus par différentes voies.

Au cours de nos essais, le début de mouvement était caractérisé par le déplacement des premiers grains de matériau, mais cette indication était, si l'on peut dire, contrôlée. En étudiant le transport solide proprement dit sur le fond, nous pouvions en effet déduire, d'après la courbe $Q$ solide en fonction des caractéristiques hydrauliques, les valeurs de celles-ci pour lesquelles le débit solide était nul ou pratiquement tel. 


\section{c) Matériaux considérés.}

Nous avons utilisé nous-mêmes deux granulométries de pierre ponce, différentes granulométries de pollopas et de sables, les densités étaient respectivement : 1,38, 1,46 et 2,65 (densité des grains effectivement transportés.

\section{d) Résultats expérimentaux.}

Nous avons porté sur le graphique figure 4, en coordonnées logarithmiques, les valeurs $d_{1}$ en fonction de $\mathrm{T}$ correspondant au démarrage des

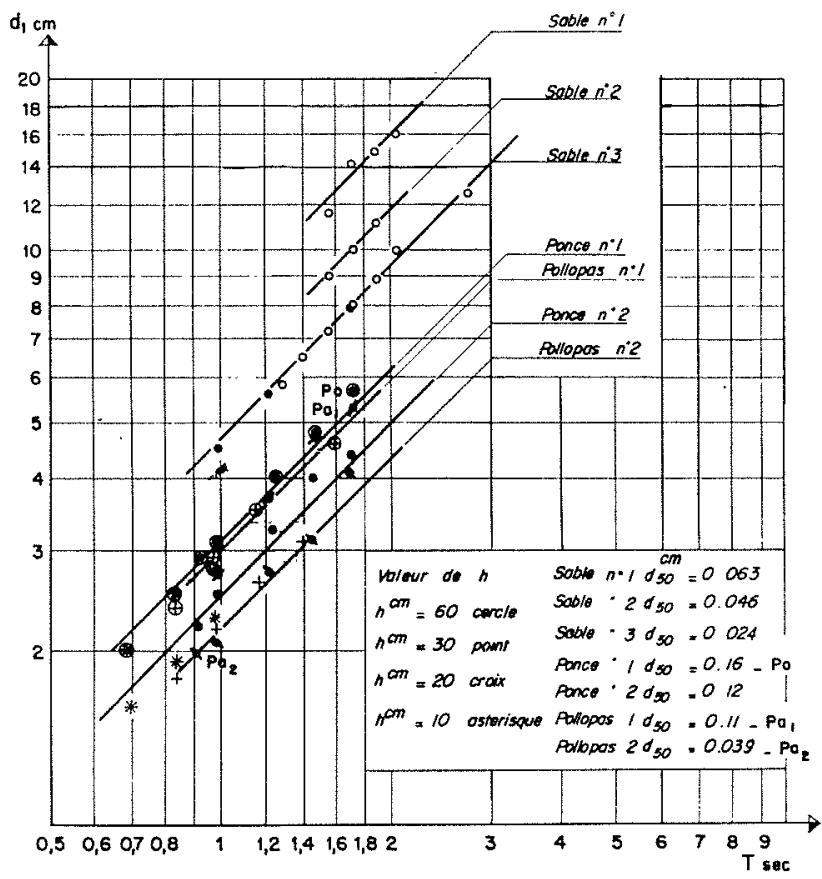

Firk. 4. - Démarrage des grains. - Relation $d_{1}=f(\mathrm{~T})$.

grains $\left(d_{1}\right.$ n'est pas la valeur mesurée de l'excursion totale d'une particule au voisinage du fond, mais la valeur théorique).

On constate que, dans le domaine considéré, $d_{1}(\mathrm{~T})$ peut s'exprimer correctement par une relation de la forme :

$$
\mathrm{T}^{-1} d_{1}=\psi=\left(\mathrm{V}_{0} / \pi\right)
$$

$\psi$ étant une fonclion dépendant uniquement du matériau et du fluide (viscosité cinématique),

Pour un matériau donné, on remarque une certaine influence de la profondeur d'eau, c'està-dire en fait de la profondeur relative $h / L$.

Cette «dispersion » peut provenir soit du fait que la valeur théorique $d_{1}$ ne désigne qu'une valeur approchée de l'excursion totale effective de la particule liquide au voisinage du fond (des éludes expérimentales ont révélé de tels écarts dépendant de $h / \mathrm{L}$ ) soit que d'une manière générale $d_{1}$ et $T$ ne suffisent pas à eux seuls à caractériser le début du mouvement des grains d'un matériau donné. (On constate par exemple que, pour un matériau et un fluide donnés, $V_{0}$ croit légèrement avec $\mathrm{T}$. Une explication de cette constatation peut être trouvée dans le fait que l'épaisseur de la couche limite croît également avec $\mathrm{T}$.

Nous verrons ultérieurement que la vitesse orbitale maximum au voisinage $\mathrm{du}$ fond :

$$
\mathrm{V}_{0}=\pi d_{1} \mathrm{~T}^{-1}
$$

(premier ordre d'approximation) n'est qu'une valeur approchée de la vitesse réelle.

\section{e) Influence des caractéristiques physiques du grain.}

Il serait sans doute illusoire de vouloir déduire de résultats trop fragmentaires une relation liant la vitesse de début de mouvement du grain à ses caractéristiques physiques.

En conséquence, nous nous bornons à présen-

\begin{tabular}{|c|c|c|c|c|}
\hline MATÉRIAU & $\begin{array}{c}d \\
\mathrm{~cm}\end{array}$ & $\begin{array}{c}\mathrm{V}_{0}+\bar{u} \\
\mathrm{~cm} / \mathrm{s}\end{array}$ & $\begin{array}{c}\mathrm{W} \\
\mathrm{cm} / \mathrm{s}\end{array}$ & $\begin{array}{c}\left(\mathrm{V}_{0}+\bar{u}\right) / \mathrm{W} \\
(\cdot)\end{array}$ \\
\hline Sable $n^{\circ} 1 \ldots$ & 0,063 & 28,50 & 9,4 & 3,03 \\
\hline Sable $n^{\circ} 2 \ldots$ & 0,046 & 20,50 & 6,0 & 3,42 \\
\hline Sable n*3. & 0,024 & 16,50 & 3,3 & 5,00 \\
\hline Sable $n^{\circ} 4$ & 0,010 & 12,50 & 0,8 & 15,60 \\
\hline Ponce $n^{*} 1$ & 0,160 & 11,00 & 8,9 & 1,24 \\
\hline Ponce $n^{\circ} 2$ & 0,120 & 8,50 & 7,3 & 1,16 \\
\hline Pollopas $n^{\circ} 1 \ldots$ & 0,110 & 10,50 & 5,6 & 1,86 \\
\hline Pollopas $n^{\circ} 2$ & 0,039 & 7,00 & 2,1 & 3,30 \\
\hline
\end{tabular}
ter quelques remarques sur nos résultats.

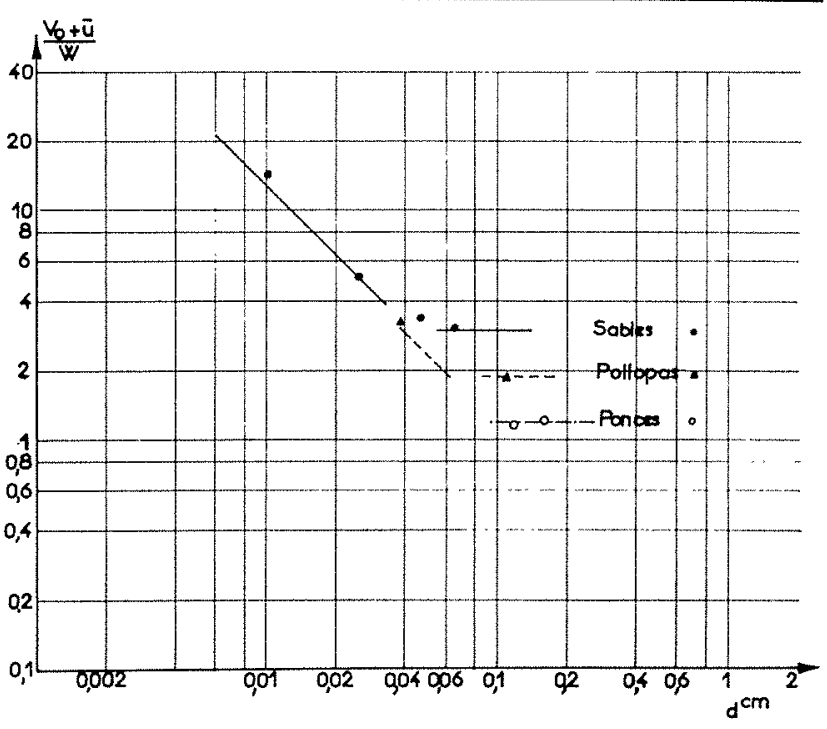

Fig. 5. - Relation :

$$
\left(\mathrm{V}_{0}+\bar{u}\right) / \mathrm{W}^{\gamma}=f(\hat{g}, d)
$$

(*) $\bar{l}$ est la vitesse moyenne d'un courant d'entrânement des particules liquides au voisinage immédiat du fond. 
A partir des résultats indiqués ci-dessus, nous avons porté sur un graphique, en coordonnées logarithmiques, $V_{0}+\pi / W$, en fonction de $d$ (fig. 5).

On constate, pour un matériau de masse spécifique donnée, l'existence d'une relation de la forme $\left(V_{0}+\ddot{u}\right) / \mathrm{W}=\mathrm{F}\left(d^{m}\right), m$ variant avee $d$. Ce résultat est à rapprocher des remarques exprimées par M. Valembois sur les résultats de M. Larras en ce qui concerne l'influence de $d / d_{0}$.

Pour des valeurs de $d / \dot{d}_{0}$ faibles, c'est-á-dire lorsque le grain de matériau, lors du début de mouvement, est pratiquement complètement enveloppé par la couche limite laminaire oscillatoire, $\left(V_{0}+\pi\right) / W$ semble ètre, pour un matériau de masse spécifique donnée et pour un fluide donné (l'eau), de la forme $\left(\mathrm{V}_{0}+i i\right) / \mathrm{W}=k / d$. Par contre, Iorsque $d / d_{0}$ atteint des valeurs telles que le grain de matériau n'est plus protégé de l'écoulement supérieur par la couche limite laminaire, soit parce que l'épaisseur de celle-ci est relativement trop faible, soit parce qu'elle est partiellement turbulente, $\left(V_{0}+u\right) / W$ tend à devenir indépendant de $d$.

Ainsi, de manière générale, pour un fluide donné, la relation entre $\left(\mathrm{V}_{0}+\ddot{u}\right) / \mathrm{W}$ et $d$ paraît dépendre de la valeur relative $d / d_{0}$, soit finalement de T. Pour un fond de sable, dans la natare, l'écoulement au voisinage du fond devant être le plus souvent turbulent, $\left(\mathrm{V}_{0}+i t\right) / \mathrm{W}$ serait indépendant de $d$, mais dépendrait légèrement de $\hat{g}$, comme l'indique la figure 5 .

\section{f) Etat de turbulence au voisinage du fond lors du démarrage des grains.}

Par analogie avec le nombre de Reynolds de "transition » de Huon Li, on peut définir un nombre de Reynolds de « démarrage » de la forme $\omega d_{1} \varepsilon / \nu$; celui-ci est en moyenne une constante pour chaque rugosité.

\section{III. - VITESSE D'ENTRAINEMENT AU VOISINAGE IMMEDIAT DU FOND : VITESSE MAXIMUM DES PARTICULES LIQUIDES AU VOISINAGE IMMÉDIAT DU FOND LORS DU DÉMARRAGE DU GRAIN}

Nous avons vu que l'expression $d \mathrm{~T}^{-1}=\mathrm{V}_{0}$ caractérisait approximativement le début de mou-

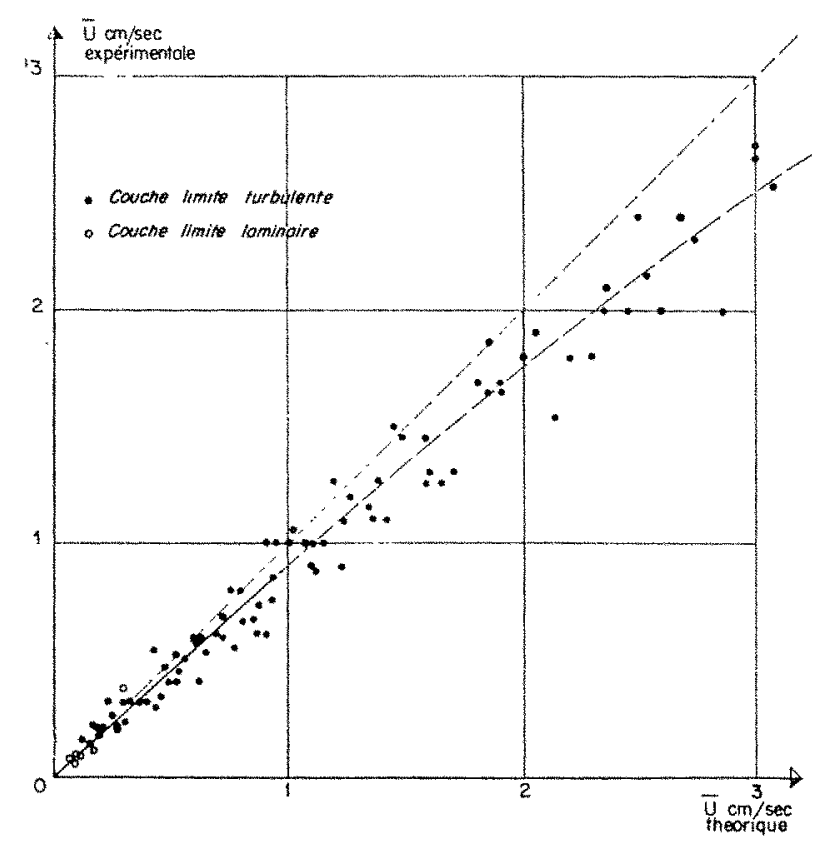

Fici, 6. - Vitesse maxima du courant de masse au voisinage du fond. - Comparaison entre valeurs expérimentales ef théoriques (Longuet-Higgins). vement des grains d'un matériau donné. En fait, la vitesse maximum résultante d'une particule liquide au voisinage immédiat du fond est légèrement différente de la valeur théorique $\mathrm{V}_{0}$ caractérisant la vitesse, dans un fluide parfait, d'une particule fluide sur le fond (premier ordre d'approximation).

Dans un calcul récent, Longuet-Higgins a montré que dans un fluide visqueux, en régime laminaire, au voisinage immédiat du fond (plus exactement dans la couche limite), une houle progressive s'accompagnait d'un courant d'entraine. ment portant dans le sens de propagation.

L'expérience confirme l'existence de ce conrant d'entrainement. On a en effet constaté, lors d'essais en canal, qu'à proximité immédiale $d u$ fond, les particules liquides progressaient en moyenne dans le sens de propagation de la houle.

On a désigné la valeur moyenne maximum de ce courant d'entraînement par $u ̈$.

Ainsi, an voisinage immédiat du fond, la vitesse des particules fluides agissant sur les grains de matériau peut être considérée comme étant la résultante de deux vitesses :

- le courant d'entrainement dont s'accompagne la houle progressive, 
- la composante pulsatoire, résultant du mouvement général d'oscillation ( ${ }^{*}$ ).

Au voisinage immédiat du fond, les valeurs maxima de ces deux composantes de la vitesse s'ajoutent algébriquement puisque toutes deux sont parallèles au fond; elles sont de plus, dans ce cas, horizontales.

\section{a) Intensité du courant d'entraînement au voisinage immédiat du fond.}

Selon les calculs de Longuet-Higgins, confirmés comme nous le verrons par l'expérience, l'in-

( ) Au premier ordre d'approximation, le mouvement apparaît symétrique; en un point donné, la vitesse d'une particule liquide dans le sens de propagation (passage d'une crête) est égale à la vitesse dans le sens contraire (passage d'un creux).

Au deuxième ordre, qui correspond mieux à la réalité, $V(t)$ pour une particule donnée n'est plus symétrique, la vitesse maximum de la particule au passage d'une crête est supérieure à la vitesse de cette même particule lors du passage d'un creux. Nous savons d'autre part qu'il peut $y$ avoir alors un courant de transport de masse.

En houle réelle et en profondeur finie, l'expérience confirme qu'à une certaine distance du fond, les orbites de houle ne sont plus symetriques. En profondeur relative $h / L$ faible et pour une houle de cambrure appréciable, on distingue nettement sur l'orbite une double asymétrie (verticale et horizontale). Sur une orbite, la courbure est plus forte à la partie supérieure qu'à la partie inférieure (fig. $7 a$ ) l'orbite n'est pas ferméc. excepté cependant à une certaine distance au-dessus du fond où la dissymetrie verticale disparait.

Celle double dissymetrie se traduit sur la courbe camelérisant la vitesse d'une particule liquide parcourant unc orbite par une allure s'écartant sevsiblement de la forme sinusoidale (fig. 7 b).

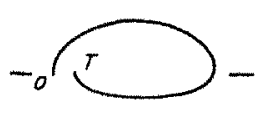

Fig. $7 a$

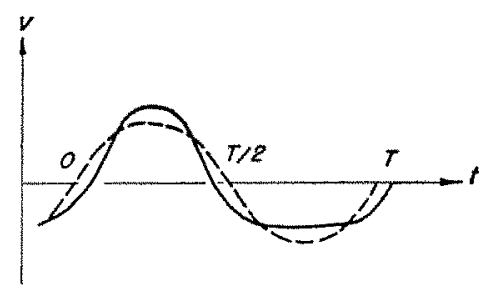

Fig. $7 b$
Cette difference sur les vitesses des particules dirigées dans le sens de propagation et dans le sens opposé tend $\dot{a}$ favoriser le transport solide vers le rivage.

Cette différence est d'autant plus marqué que $h / L$ est faible, esest-àdire sur une profondeur donnée que $L$ est grand. A la limite, la houle est d'ailleurs couramment. rapproche de l'onde solitaite.

Aussi, sur les fonds fribles, si l'on considère l'effet global du courant d'entrainement et de la dissymetrie. des vitesses orbitales dans le sens de propagation et dans le sens opposé, on est conduit à penser que les houles longues, peu cambrées, sont susceptibles de provoquer d'importants transports solides vers le vivage.

Ceci tendrait a expliquer que les houles de «bean temps $\gg$ (longues et plates) incapables de donner naissance a de forts rip-currents et a de forts underflow (c'est-à-dire en fait de suspension) tendent particulie. rement à cngraisser les plages. tensité maximum de ce courant d'entrainement dans une houle progressive est donnée par une expression de la forme (page 568) :

$$
\bar{u}_{\max }=\frac{1,376 \mathrm{~T}}{\mathrm{~L}}\left(\frac{2 \pi a}{\mathrm{~T}}\right)^{2} \frac{1}{\operatorname{sh}^{2} 2 \pi h / \mathrm{L}}
$$

c'est-à-dìre :

$$
\vec{u}_{\operatorname{mix}}=\frac{1,376 \mathrm{~T}}{\mathrm{~L}} \mathrm{~V}_{0}^{2}{ }_{\max }
$$

$\mathrm{V}_{0 \max }$ étant la valeur théorique maximum de la composante horizontale de la vitesse orbitale (premier ordre, fluide parfait).

Les mesures du courant d'entrainement au voisinage immédiat du fond effectuées au cours de nos essais confirment cette expression avec une assez bonne approximation. A titre d'indication, nous avons porté ces résultats sur la figure 6 ; on constate en général que la différence entre la vitesse mesurée et la vitesse théorique donnée par l'expression de Longuet-Higgins est faible, quand l'écoulement dans la couche limite est laminaire (domaine de validité de la théorie). Cette divergence s'accroît lorsque la turbulence se développe.

\section{b) Vitesse maximum des particules liquides au voisinage immédiat du fond lors du démarrage des grains.}

La résultante de la vitesse des particules liquides au voisinage du fond a finalement l'allure représentée figure $7 c$.

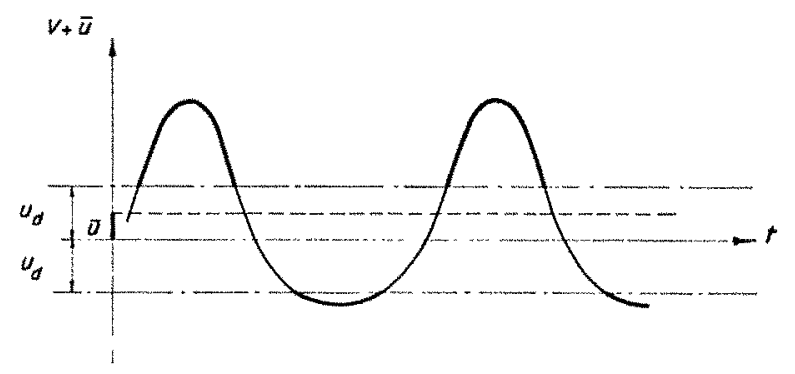

Fig. To

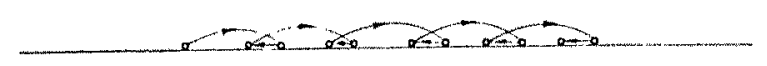

Fig. $7 d$

Si $\mathrm{U}_{d}$ caractérise la vitesse de débul de mouvement d'un grain donné, le grain commencera à se déplacer lorsque la valeur maximum de la vilesse absolue, par rapport a un grain de matériau immobile, d'une particule liquide at voisinage immédiat du fond sera au moins égale à $U_{t}$.

Au deuxième ordre (fluide parfait), au voisinage immédiat du fond on peut considérer que ceci est vrai à la limite supérieure de la couche 
limite, la vitesse maximum d'une particule liquide est de la forme :

$V_{0} \pm$ [ un terme au second ordre de la forme $\left.\mathrm{V}_{0}^{2} f(h, \mathrm{~L})\right]$

A ce terme "périodique », pour un fluide visqueux s'ajoute la vitesse $\bar{u}$ du courant d'entraìnement de Longuet-Higgins $\left(2^{\mathrm{e}}\right.$ ordre dans la couche limite).
On constate que la valeur $V_{0}+\bar{U}$ correspondant au début de mouvement des grains d'un matériau donné est pratiquement constante, quelles que soient les valeurs relatives des facteurs ayant donné naissance à ces vitesses. (Nous avons vu, toutefois, que $V_{0}$ croissait légèrement avec $\mathbf{T}$ et nous avons expliqué cette croissance par l'accroissement de l'épaisseur de la couche limite.)

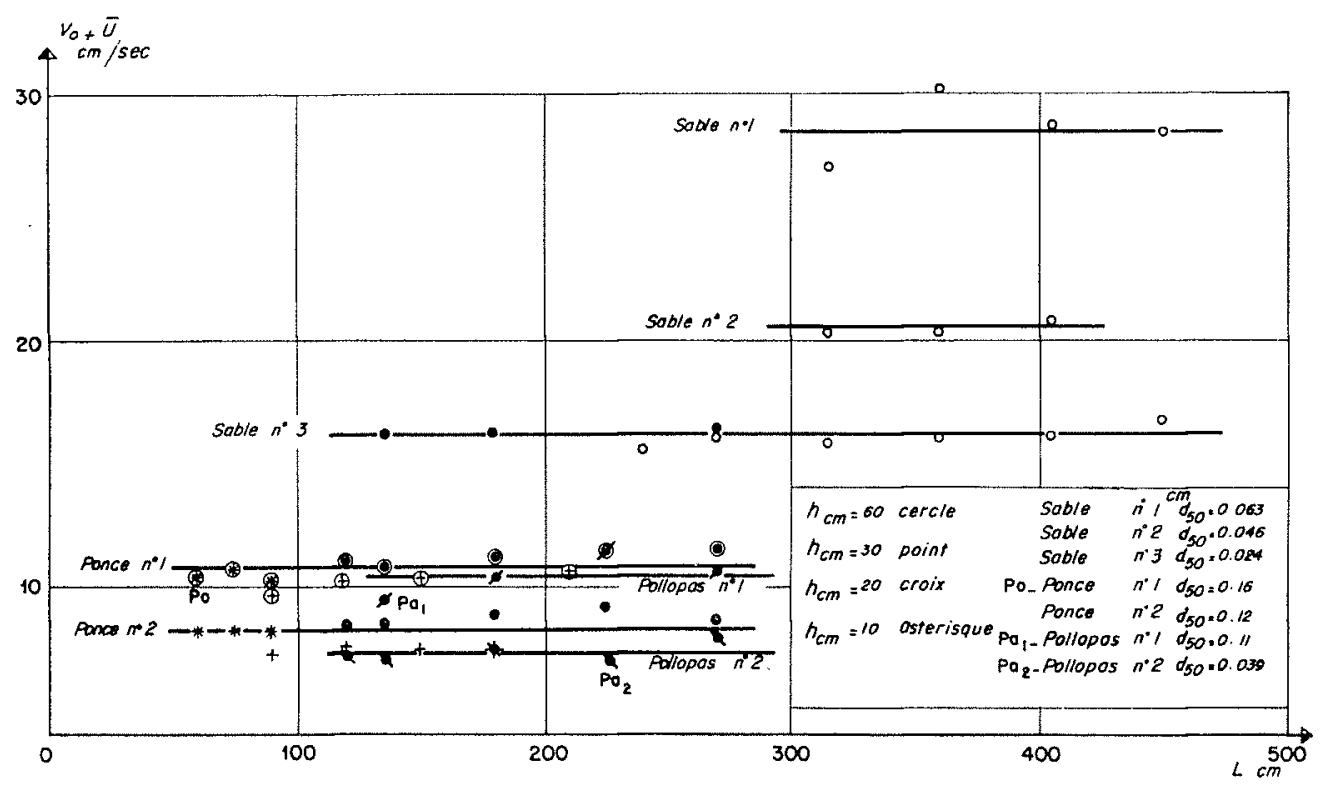

Fus. 8. - Vitesse de démarrage des grains

Relation $\mathrm{V}_{\mathrm{u}}+\mathrm{U}=f(\mathrm{~L})$.

Lors du début de mouvement, nous pouvons négliger le terme au second ordre entre crochets et écrire que le début de mouvement du grain se produit lorsque :

$$
\mathrm{U}_{d}=\bar{u}+\mathrm{V}_{0}
$$

Nous ayons calculé pour les divers matériaux considérés les valeurs respectives de $V_{0}, \bar{u}$ et $\left(V_{0}+\bar{u}\right)$; le graphique figure 8 donne les valeurs de $\bar{u}+V_{0}$ en fonction de $L$ pour sept matériaux.
En outre, étant donné la forme de la vitesse résultante au voisinage immédiat du fond, la trajectoire d'un grain de matériau dans le sens de propagation de la houle sera plus grande que dans le sens contraire. Ayant démarré, le grain se déplacera donc par bonds successifs avant et arrière, les bonds en avant étant plus amples que les bonds en arrière (fig. $7 d$ ). C'est-à-dire que finalement, sur le fond horizontal, le grain de matériau progressera dans le sens de propagation de la houle.

\section{IV. - LE TRANSPORT DES MATERIAUX SOUS L'INFLUENCE DE LA HOULE SEULE}

Par le fait même qu'au voisinage immédiat du fond en particulier, la résultante des vitesses dans le sens de propagation est supérieure en intensité à la résultante des vitesses dans le sens opposé, le début de mouvement des grains s'accompagne d'un transport solide réel.

\section{a) Courant de transport de masse dans une houle monopériodique pure.}

Pour une houle à deux dimensions, on constate, au cours d'essais en canal, qui le mouvement d'oscillation des particules s'accompagne, dans la masse fluide, d'un mouvement général. 
La distribution verticale des vitesses de ce courant de transport présente les caractéristiques suivantes $\left(^{*}\right)$ :

- A proximité de la surface, le courant porte dans le sens de la propagation de la houle; on a observé expérimentalement, pour les faibles valeurs de $h / L$, un courant portant en sens inverse.

- A proximité du fond, le courant porte toujours dans le sens de propagation; il est particulièrement marqué dans une zone de faible épaisseur, au voisinage immédiat du fond (courant d'entrainement).

- Entre ces deux zones, le courant est de sens opposé; en canal fermé, le débit sur une verticale étant nul, les débits dans les deux sens sont égaux.

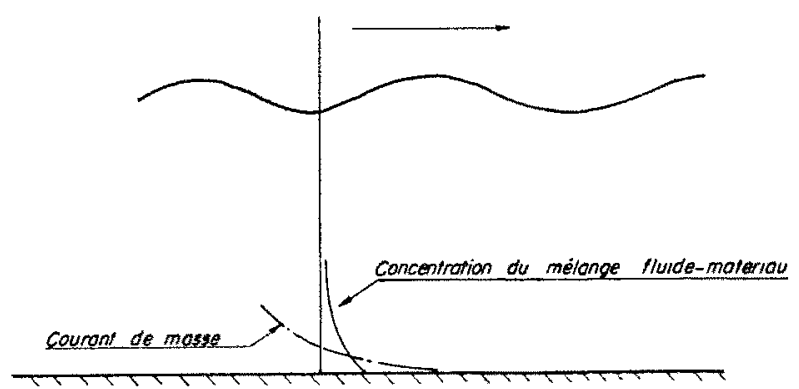

fig. 9 a.
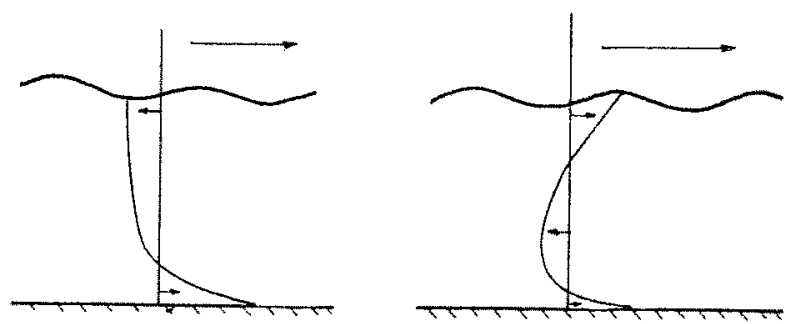

$\frac{h}{L}$ toible

fig. $9 \mathrm{~b}$.

$$
\frac{h}{L} \text { grons }
$$

On indique figure $9 b$, de manière schématique, deux répartitions-types de ce courant observées au cours d'essais en canal fermé.

\section{b) Inversion du transport solide global sur fond horizontal.}

Ainsi, de manière générale, on constate en canal d'essais que pour une houle pratiquement

(*) Ce courant de transport de masse apparait dans les équations de la houle, à partir du $2^{\circ}$ ordre. Toutefois, en fluide parfait, la distribution vertieale de la vitesse est arbitraire (fluide parfait).

Des courants de cette nature ont été mis en évidence en canal expérimental notamment par Caligny 1878 , Mitchim 1939, Bagnold 1947, King 1948, Laboratoire Dauphinois d'Hydraulique 1949.

L'existence de tels courants en mer parait très vraìsemblable en houle suffisamment régulière. pure sur un fond horizontal, le transport solide est dirigé dans le sens de propagation de la houle.

Dans certaines conditions toutefois, le débit solide global, c'est-à-dire le débit solide, non plus au voisinage immédiat du fond, mais intégré sur une verticale, commence à croître avec l'amplitude de la houle, passe par un maximum, décrôt, puis, dans certaines circonstances, peut s'inverser.

Ce phénomène se produit dans le canal d'essais lorsque la turbulence étant grande, la couche de matériau en suspension près du fond atteint les couches intermédiaires du fluide an sein desquelles le courant de transport est dirigé vers le large.

Des phénomènes de même nature apparaissent encore lorsque la houle n'est plus pure, c'està-dire lorsqu'on a affaire à un clapotis partiel; la distribution verticale du courant de transport apparait d'ailleurs particulièrement sensible à la «pureté de la houle».

Ainsi, selon les valeurs relatives du débit solide dirigé dans le sens de propagation et dans le sens opposé, e'est-à-dire selon la répartition $u_{z}$ (résultante de la vitesse) et $c_{m}(z)$ (concentration moyenne en matériau), le débit solide global pourra être dirigé dans un sens on dans l'autre.

Les fonctions $\bar{u}$ et $c_{m}$ présentent généralement dans la zone intéressée l'allure figurée en $9 a$.

On conçoit l'intérêt que présentent ces résultats qui, avec les courants de densité, permettent de donner une explication apparemment satisfaisante à l'existence, dans un profil, de mouvements solides dirigés vers le large.

\section{c) Recherche d'une loi empirique à partix des résultats expérimentaux.}

Considérant l'action de la houle sur un fond horizontal, nous avons, au cours de cet exposé, mis en évidence plusieurs paramètres qui ont une influence directe sur le mouvement solide : la vitesse orbitale des particules, le courant d'en traînement, la turbulence, etc.

Suffisamment au-delà du déferlement, vers le large, la plupart de ces paramètres sont en général à eux seuls incapables de provoquer un transport de matériau, tout au plus le favorisent-ils; en l'absence de courant marin ordinaire, on pourrait penser que seul le courant d'entraînement transporte effectivement les matériaux. Il en sera généralement autrement dans les zones peu prom fondes, la vitesse orbitale devenant sensiblement dissymétrique, ou dans les zones très peu profondes où l'onde d'oscillation peut prendre l'aspect de l'onde de translation faisant apparaître, au voisinage du fond notamment, une différence de vitesses très notable lors du passage de la crête ou du creux de la vague. 
Ceci étant, on peut s'attendre à ce que l'on ne puisse trouver - en particulier dans les zones considérées comme peu profondes, eu égard à la longueur d'onde de la houle - une relation simple liant le transport solide aux caractéristiques hydrauliques d'une part et aux caractéristiques du matériau, d'autre part.

\section{RECHERCHE D'UNE RELATION $\left(Q_{\text {solide }}=f\left(V_{0}\right)\right.$.}

Nous avons vu que l'expression $d_{1} \mathbf{T}^{-1}$, c'està-dire $\mathrm{V}_{0}$ (vitesse orbitale maximum, $1^{\text {er }}$ ordre) était un paramètre fondamental caractérisant le début d'un mouvement d'un matériel donné. $V_{0}$ joue un ròle analogue vis-à-vis du transport so-
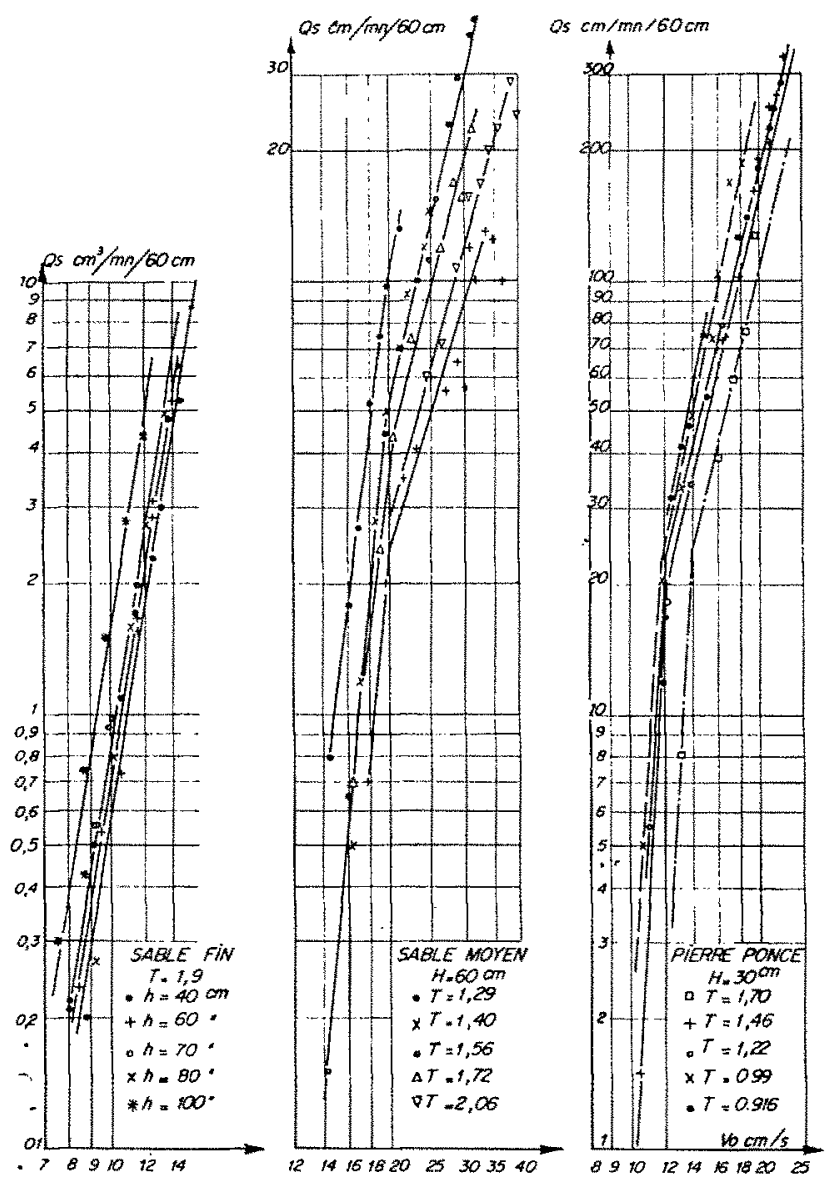

Fig. 10. - Transport solide provoque par la houle sur un fond horizontal. - Relation $Q_{\text {sol }}=f\left(\mathrm{Vo}_{0}, h_{h}, \mathrm{~T}\right)$ pour trois matériaux.

lide provoqué par l'action de la houle seule.

Pour illustrer le rôle principal de ce facteur, nous avons porté, à titre d'exemple, sur le graphique figure 10 , la relation $\mathrm{Q}_{\text {solide }}=f\left(\mathrm{~V}_{0}\right)$ pour trois matériaux: un sable de granulométrie moyenne, un sable très fin et de la pierre ponce. Nous avons volontairement choisi deux matériaux donnant des rides sur le fond et, à des degrés divers, de la suspension (les sables), et un autre matériau ne donnant pratiquement, tout au moins dans la gamme - pourtant assez large - des conditions hydrauliques considérées, ni ride ni suspension (la pierre ponce).

En observant ces résultats, on constate :

1. Outre $V_{0}$, le débit solide dépend également, pour un matériau et un fluide donnés, de la profondeur d'eau $h$ et de la période T (ou longueur d'onde L) de la houle. Pour une valeur donnée de $V_{0}, Q_{\text {solide }}$ croît lorsque $h$ croit et lorsque $\mathrm{T}$ décroît (ce résultat, à première vue paradoxal, met en relief l'influence de $V_{0}$ ).

2. Pour chaque matériau (L et $h$ étant donnés), $Q_{\text {solide }}$ s'exprime par une relation de la forme $\mathrm{Q}_{0}=\mathrm{K} \mathrm{V}_{0} . m$ n'est pas indépendant de $\mathrm{V}_{0}$; on constate, quelles que soient les valeurs de $L$ ou de $h$, que $m$ change pour une valeur remarquable de $V_{0}$, c'est-à-dire pour une certaine valeur d'un nombre de Reynolds $2 \mathrm{~V}_{0} \mathrm{\varepsilon} / \mathrm{v}$ (ou $\left.\omega d_{1} \varepsilon v\right)$ susceptible de caractériser la turbulence autour du grain.

Les phénomènes étant les mêmes pour deux matériaux, l'un donnant des rides et de la suspension, lautre ne donnant ni ride ni suspension, on peut être tenté de conclure que la turbulence au voisinage du fond joue un rôle important au cours des premières phases de transport solide.

En fait, il semble que l'on puisse distinguer trois stades dans le transport solide provoqué par la houle :

$1^{\text {er }}$ STADE : faible turbulence au voisinage du fond, le transport se produit uniquement par charriage sur le fond.

$2^{\circ}$ STADE : turbulence moyenne, le transport se manifeste principalement par charriage et suspension, sans toutefois que celle-ci atteigne audessus $\mathrm{du}$ fond les couches fluides dans lesquelles se manifeste la composante du courant de masse dirigé dans le sens opposé au sens de propagation.

$3^{e}$ STADE : forte turbulence, le transport se manifeste par charriage et suspension sur une hauteur relativement importante au-dessus $d u$ fond. Nous avons vu que, dans ce cas, le transport solide global n'est pas nécessairement important.

Excepté pendant les tempêtes, durant lesquclles la suspension peut atteindre, au-dessus du fond, une hauteur telle qu'elle se manifeste dans la zone où le courant de masse est opposé au sens de propagation de la houle, c'est la situation intermédiaire que l'on doit rencontrer le plus fréquemment dans la réalité.

La caractéristique essentielle du transport pendant le premicr et le sccond stade est que le dé- 
bit solide proprement dit varie très rapidement avec $V_{0}$, pour le sable fin par exemple dans la gamme de nos observations, correspondant à ce que nous avons appelé le deuxième stade :

\section{$\mathrm{Q}_{0} \# \mathrm{KV}_{0}{ }^{6}$}

$\left(Q_{0}\right.$ varie alors peu avec $h$ et $\mathrm{T}$ pour un $\mathrm{V}_{0}$ donné).

\section{V. - INFLUENCE DIRECTE DE LA HOULE SUR LES FONDS}

II ressorl nettement de l'étude précédente qu'au voisinage du fond, la houle transporte les matériaux dans le sens de sa propagation, c'est-à-dire pratiquement vers la còte. Dans cette zone, le transport solide dans le sens de propagation est en général, pour une houle donnée, d'autant plus important que la profondeur est faible, le matériau plus fin et plus léger. On pourrait ainsi être tenté de croire que les matériaux s'acheminent indéfiniment vers la côte. En fait, c'est bien souvent l'inverse qui se produit, les matériaux érodés au continent se perdant vers le large.

Nous avons vu, au cours de cette étude, que le débit solide global sur une verticale pouvait en effet s'annuler, parfois même s'inverser; nous avons attribué cette inversion du transport solide au rôle prépondérant que peut prendre, dans certaines circonstances, la composante du courant de transport dirigée vers le large. Mais il y a, croyons-nous, d'autres raisons encore. (Mise à part l'influence évidente de la gravité, en particulier au voisinage du rivage où les pentes de fond sont parfois importantes).

Nous citions, au début de cette note, l'action de la houle sur les fonds quant à son pouvoir de développer des courants de densité qui entraîneront vers le large les particules fines.

Nous pourrions y ajouter l'irrégularité de la houle naturelle, notamment de la houle de tempête; nous avons constaté au cours de nos essais combien l'allure de courant de transport, par exemple, pouvait être affectée par une réflexion partielle on une irrégularité dans la période.

Les mouvements de matériau vers le large peuvent être encore favorisés par l'action, parfois violente, du vent en surface; celui-ci peut en effet, au cours d'une tempête en particulier, provoquer un courant d'intensité notabie portant en surface vers la côte et déplaçant au voisinage du fond les matériaux vers le large.

Les courants, parfois même violents, qui se manifestent dans la zone de déferlement des vagues jouent également un rôle non négligeable sur les mouvements solides vers le large, ne serait-ce qu'en favorisant le développement des courants de densité. II y a tout lieu de penser qu'en raison même du principe de la continuité, ces « courants littoraux » s'intègrent intimement dans le système, sur lequel nous avons plus par- ticulièrement insisté ici, des courants se manitestant au large de la zone de defferlement.

\section{TrANSPORT SOLDE VERS LE LARGE PAR COURANT DE DENSITÉ}

A l'image des écoulements par gravité, l'expérience montre que la houle produit des courants de densité.

Pendant une période forte houle (tempête, par exemple), une quantité considérable de matériau peut être arrachée du fond et mise en suspension dans la zone de déferlement. Ce phénomène est évidemment d'autant plus prononcé que les matériaux dont il s'agit sont plus fins et le dépôt de la plage attaquée par la houle conslitué de matériaux plus récents. (Les courants de densité sont principalement formés par des particules solides dont la dimension est voisine ou inférieure à $50 \mu$, mais ceux-ei sont, de par leur nature, capables d'entrainer des particules plus grosses.)

Par suite de leur densité moyenne relativement plus grande que celle du milieu qui les entoure, les eaux chargées plongent vers le fond, puis s'écoulent vers les régions profondes.

Des expériences effectuées en canal à houle montrent que l'écoulement de la masse turbide vers le fond s'accompagne d'un contre-conrant d'eau claire dans les couches fluides supérieures, les eaux de surface restent done claires.

S'agissant de la houle, ce phénomène est compliqué par la présence de courants liés à l'existence même de la houle naturelle qui se manirestent jusque dans la zone de déferlement..

Néanmoins, il est probable que dans la réalité les courants de houle ne jouent vis-à-vis des courants turbides qu'un rôle secondaire. Les courants de densité vers le large se développent en effet particulièrement vers la fin de la tempête, c'est-à-dire lorsque les courants liés à la houle disparaissent progressivement. Au plus, ces courants peuvent-ils retarder le phénomène d'écouIement du courant de densité précisément par le fait qu'au voisinage du fond, ils portent en général vers la rive et s'opposent ainsi directement au développement du courant de densité.

On assiste done vraisemblablement pendant la période d'extinction de la tempête, dans les couches proches du fond, à une prédominance pro- 
gressive du courant de densité sur le courant de masse.

Il est également probable qu'au cours de la tempête, une certaine quantité de particules parmi les plus fines puisse gagner le large dans la couche liquide intermédiaire au sein de laquelle se manifeste la composante du courant de transport de masse dirigée vers le large.

Enfin, on ne s'étonnera pas de retrouver à faible distance du rivage, lorsqu'on s'éloigne de celui-ci vers le large, une discontinuité relativement franche entre eaux colorées et eaux claires. En effet, le contre-courant du courant de densité, le courant de vent et le courant de transport de masse de la houle ont tendance à apporter en surface les eaux claires du large vers la côte,

Après une tempête, la turbulence dans la zone de déferlement étant progressivement décroissante, le flot solide du courant de densité sera lui-même décroissant, probablement avec un certain retard. Le courant de densité naissant luimême, sans doute dès le début de la tempête, il paraît raisonnable de donner à la courbe du débit solide, entraîné vers le large par le courant de densité en fonction du temps, l'allure schématisée (fig. 11).

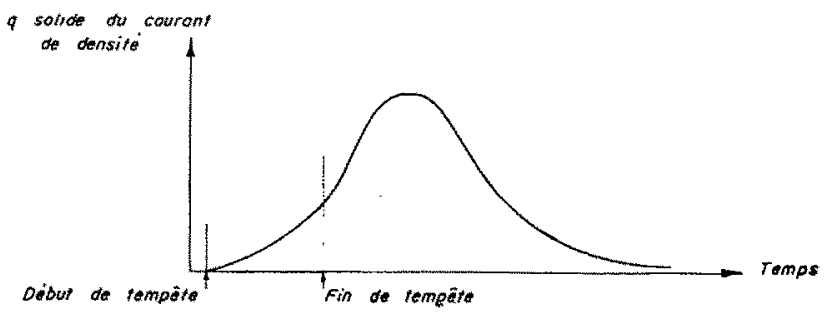

Fig. 11. - Mouvement de matériau vers le large par courant de densité.

Ceci explique que l'on rencontre au large, sur le fond de la mer, des matériaux de plus en plus fins. Les courants de densité, l'extinction progressive de la turbulence... contribuent, en effet, à ce que les matériaux se déposent d'autant plus loin du rivage qu'ils sont plus fins. II ressort, corrélativement, que, sur les plages, l'on trouve relativement peu de particules très fines, après des périodes de mauvais temps notamment.

\section{I S C US SION}

Président: M. DE Rouvrlue

M. le Président remercie M. VwaEnT d'avoir exposé sa communication dans le minimum de temps, et souhaiterait que les membres puissent méditer longuement le mémoire avant de le discuter.

M. le Président, qui a pu prendre connaissance du mémoire avant la séance, fait les remarques ci-après :

$1^{\circ}$ Dans les «généralités», le problème du transport solide sur le fond de la mer, au large de la zone de déferlement, lui rappelle un travail ancien des hydrographes sur la baic de Scine, qui tendait a conclure que la moitié environ de l'apport en Seine des matériaux dont l'origine restait inexpliquée, (l'autre moitié provenant de la côte du Calvados) était arrachée aux fonds de Ja baic, bien que la profondeur de 16 mètres environ que présentait celle-ci, semblât ne pas devoir être atteinte par les lames de 4 à $5 \mathrm{~m}$ qu'on rencontre comme maxima an milien de l'estuaire.

$2^{\circ}$ Dans le démarrage des grains, il $\mathrm{y}$ a certainement une part de hasard, plus grande dans la nature qu'en laboratoire; cela dépend de la forme, de la densite et de l'imbrication des grains. MM. VINCENT et RuLLLAN ont très bien essayé de définir une base de démarrage en fonction de ces éléments.

$3^{\circ}$ L'hypothèse, émise dans le mémoire, d'un « courant de houle», qui existerait au fond de la mer et dans le sens de la houle, avait déjà été envisagée pour expliquer la dérive des bateaux ou flotteurs vers le rivage: dans une baie abritée par une pointe, le courant peut s'expliquer par l'abaissement de la hauteur de la houle lorsqu'on s'approche de la pointe; sur une cote droite, il se peut qu'il existe, mais. il n'a aucun fondement théorique jus. qu'à prêsent.

4" Les matériaux «arrachés au rivage» le sont sur" tout par les très grandes tempêtes, mais cela ne se produit pas toujours; et, notamment au déferlement, ils sont «apportés» au rivage. $11 \mathrm{y}$ a aussi une région où les mam tériaux sont stables. Le sens des mouvements dépend de la profondeur. L'Italien Cornaglia avait présenté à ce sujet une théorie qui n'est peut-être pas absolument vraie, mais il faudrat expliquer en quoi on la modifie.

M. VINCENT répond à M. le Président :

1. Les sables de la baie de Seine peuvent avoir été mis en mouvement par la houle, grâce à son action propre, puis transportés par les courants ordinaires qui ne sont pas négligeables et règnent jusqu'au fond.

$2^{\circ}$ La part du hasard est en effet sensiblement réduite par le choix de notre critère de démarrage; celui-ci étant lié, comme on l'a vu, au transport solide proprement dit.

$3^{\circ}$ La distinction que loon fait dans le mémoire entre le courant d'entran̂nement (au voisinage immédiat du fond) et le courant de transport (dans la masse fluide) n'est pas uniquement de pure forme. L'existence, dans la nature, d'un courant d'entraimement portant, au voisinage du fond, toujours dans le sens de la houle nous paraît devoir être bien moins contestée que celle du courant de transport, dont la distribution verticale peut être, en théorie, choisie arbitrairement. L'observation de ce conrant en canal expérimental ne suffit pas évidemment a prouver son existence dans la nature.

$4^{\circ}$ Au voisinage de la zone de déferlement, la pente du fond joue un rôle important sur le mouvement des grains de matériau : elle contrebalance l'influence de la houle. Une houle allongée, pea turbulente dans l'ensemble, possède un pouvoir plus grand pour remonter les matériaux vers le rivage qu'une houle plus cambrée; toutes choses égales par ailleurs, le profil d'équilibre de cette 
partie de la plage sera plus accore en houle allongée. L'existence d'une «ligne neutre» comme le suggère la thèse de l'ingénieur italien Comaglia no nous parait pas spécialement s'accorder aux résultats présentés dans notre mémoire. Il se produit certes tantôt des mouvements de matériaux vers le rivage, tantôt vers le large, selon les caractéristiques des vagues, mais il paraît peu vraisemblable que de tels mouvements dans un profil existent simultanément de manière systématique.

M. Lhermitrs indique toutefois que M. Bouasse signale dans son livre (1) un écrit de Bertrand de Calligny, d'après lequel il aurait été constaté, dans le fond de l'océan, un courant portant dans le sens de la propagation de la houle.

En réponse à une question de M. Lhenmitte, M. Vincex'T répond qu'en effet, la houle donne plus de turbulence sur le fond que le laissaient prévoir les essais de Huon-Li, mais que cela est particulièrement vrai pour un fond perméable. Dans ie cas d'un fond lisse, les essais ne sont pas suffisamment poussés actuellement, mais M. VINCENT pense pouvoir donner des renseignements a ce sujet dans le texte définitif.

M. LARRAs et M. LHERMITTE, très intéressés par cette communication, proposeni, vu l'heure tardive et l'importance du sujet, de reporter la discussion de la question a un petit comité qui pourrait se rénnir après que MM. Vincent et Rublian auraient pu ajouter à leur mémoire de nouvelles indications numériques.

Cette proposition est adoptée.

\title{
SUITE DE LA DISCUSSION (Séance du 22 novembre 1957)
}

\author{
Président : M. Barrillon
}

M. le Président organise Ia diseussion suivant les six paragraphes des conclusions figurant au début du mémoire de MM. Vincent et RUelaA.

$1^{\circ}$ Les résultats donnés par Huon-Li concernant l'apparition de la turbulence dans la couche oscillatoire donnent trop d'importance au laminaire. $V_{0}$ (vitesse. orbitale maximum) et $\varepsilon$ (rugosité) sont les facteurs principaux caractérisant la transition.

M. Vincent précise que la turbulence de la houle expérimentale au voisinage du fond $y$ est supposée défnic qualitativement par des formes en volute prises par des colorants, mais non mesurée.

En réponse à une question de $M$. oe Rovvrlle, sur l'emplacement exact de la couche limite laminare oseillatoire, M. VINCENT indique que cette couche est caractérisée comme étant le siège d'un gradient de vitesse très important, allant de 0 sur le fond à une valeur $V_{o}$ à la partic supérieure de la couche. L'épaisseur de la couche n'a pas été mesurée, mais sa valeur théorique est donnée par une expression indiquée dans le mémoire.

M. le Président conclut qu`il s'agit d'un sol lisse.

Pour M. Valemisors, l'idée émanant du mémoire de MM. Vincent et Ruellan, que le critérium du passage du laminaire au turbulent est différent suivant que l'on a un mouvement $\mathrm{du}$ fond relatif à l'eau immobile (HuoN-LI) ou un mouvement de l'eau relatif au fond immobile (VINCENT et RumLLaN), va à l'encontre de la conclusion, contenu dans ce demier mémoire, à savoir que si l'on arrivait a réaliser le régime lisse, on trouverait une valeur de la transition sensiblement égale au nombre sans dimension 800, qui est aussi celle trouvée par Huon-Li.

M. Vincent estime que la différence d'interprétation vient de la difficulté de caractériser physiquement et quantitativement un fond lisse. En indiquant que le nombre de Reynolds correspondant à un fond hydrauliquement lisse n'aurait pas été inférieur $\dot{a} 800$, il voulait exprimer qu'il devait être vraisemblablement supérieur à cette valeur.

M. Valembors pense que cela tient plutôt à la difficulté de caractériser le passage du laminaire au turbulent qui, d'ailleurs, dans les expériences de Huon-Li sur fond rugueux, tendrait à des résultats un pea en contradiction avec ce qu'on pourrait attendre theoriquement d'une ex-

(1) Houles, rides, seiches ct marés (Delagrave, éditeur). périence de ce genre : il semble en effet, qu'en régime rugueux, ce critérium devrait être fonction du rapport de l'amplitude $d_{1}$ du monvement à l'épaisseur de la couche, et non du rapport: $V_{0} d_{0} / \gamma$, qui, pour des rugosités, n'a pas une signification bien claire.

M. Laenumte pense qu'il importe de bien préciser l'intérêt et les limites des expériences qui ont été faites a l'université de Berkeley par le Professeur Einstein et ses élèves : Huon-Li, Madhay Nanohar, et, tout récemment, Kalkanis. Les travaux de cette équipe, publiés dans le «Beach Erosion Board », sont basés sur l'observation d'un fond oscillant périodiquement au-dessous d'une masse de nuide immobile. Ce mode opératoire a permis d'obtenir des oscillations relatives près $d n$ fond, de lordre de grandeur de celles des honles naturelles, ce qui est inpossible en laboratoire.

Ces essais sont done interessants en ee qu'ils peurent permettre d'extrapoler les résultats de laboratoire aux houles naturelles lorsque l'expérimentation en laboratoire aura justifie les résultats obtenus par la méthode de Berkeley. Or, les expériences de laboratoire qui ont été effectuées depuis la publication de ces travaux ont montré que les résultats ainsi obtenus ne devaient s'appliquer. qu'avec de très profondes réserves aux houles réelles de laboratoire. Des causes importantes modifient profondement les résultats, et l'on peut en particulier citer parmi ces causes :

- Inlluence très différente des forees dinertic;

- Différence de gradient de pression longitudimale;

- Périodicité dans l'espace et dans le temps du mouvement de la houle, alors que l'oscillation du fond est un phénomène uniquement périodique dans le temps.

Ceci explique les écarts, par rapport aux résultats de Huon-Li, constatés au cours des expériences effectuées par: M. VINCENT, écarts que nous avons nous-mêmes observés dans les expériences effectuées au Laboratoire Central d'Hydraulique de France. En particulicr, la turbulence apparait beaucoup plus tot dans les houles naturelles que lors de l'oseillation du fond du canal, et il semble que ce résultat doive être lié a la «rigidité » du mouvement périodique dans l'espace ef dans le temps, qui s'accommode diffeilement d'une parol horizontale.

M. Laermite ajoute que la limite de stabilité de la couche limite laminaire est extrêmement importante et rejoint le probleme de l'amortissement des houles dont MM. Miche et Biesel ont étudié les raisons profondes et les conséquences qui en découlent, dans de récents articles publiés dans Ia Honille Rlanche. Enfin, M. LuEnurtw 
signale le point suivant, qui s'attache à la nature réelle de la couche limite des houles de laboratoire et qui revêt une certaine importance: les formules classiques de la houle omettent d'écrire que la vitesse longitudinale dn fluide près de la paroi est nulle; il est possible, ainsi que l'a montré M. Biesel, de tenir compte dans les équations de la houle d'une vitesse nulle près de la paroi, mais on peut se demander, étant donné l'individualité propre de la couche limite, sí ce nouveau schéma représente plus fidèlement le monvement du fluide sain au-dessus de la couche limite. Certaines observations, concernant précisément le dèveloppement des couches limites - lié au caractère propre qui lui est domné par suite de sa double périodicité dans le temps et dans l'espace - incitérajent à admettre que les équations classiques, négligeant d'écrire la nullité de la vitesse longitudinale près de la paroi, rendent compte d'une façon très satisfaisante des mouvements du fluide sain, e'est-à-dire en dehors de ta couche limite.

M. le Président confirme que l'homologue absolu de la paroi oscillante n'est pas la houle, mais un mouvement par tranches dans lequel il y a périodicité dans l'espace et dans le temps, comme dans les propagations de marée; ce mouvement s'atténue au fur et à mesure que l'on s'élève en s'éloignant du fond, alors que le mouvement. de la houle est celui d'une masse d'eau oscillant d'un bloc.

M. Valembors pense que l'on peut considerer l'écoulement de la houle au voisinage du fond, e'est-à-dire dans la partie qui intéresse la turbulence, comme un écoulement par tranches, done analogue au mouvenent de la marée.

M. Vincrer indique que, dans certaines conditions, la turbulence prenant naissance au voisinage $d u$ fond peut contaminer les couches fluides portées par la couche limite turbulente et signale que la courbe de la figure 2 donne l'épaisseur des nuages colorés, notée en canal d'essais.

M. LHEnMtTe croit qu'il est très difficile de caractériser la turbulence, car, faute d'appareil permettant de la mesurer dans l'eau, on est réduit à des observations très subjectives.

M. le Président voudrail qu'on ne parle pas de turbulence sans indiquer quel genre de turbulence on envisage : limite d'un nuage, mesure d'un chiffre et méthode de mesure.

M. VALEmors indique que l'on peut, seulement, mesurer les voiles tangentiels sur la paroi et définit lapparition de la turbulence par le point de décrochage.

M. Stapel indique que le Laboratoire de Delfit (Hollande) a mis an point un instrument mesurant les changements de vitesse et permettant de caractériser le degré de turbulence par l'importance du changement de vitesse, mais cet instrument ne permet pas encore de donner un chiffe (1)

M. Ie Président fait remarquer que la fluctuation et la turbulence sont deux choses tout à fait différentes: dans le cas prècité de la masse d'eau se déplaçant sur un fond, il $y$ a fluctuation.

M. Montagné indique que l'on peut se faire une idée de Ia valeur relative de la turbulence isotrope dans un canal dont la vitesse moyenne $U$ est par exemple de $1 \mathrm{~m} / \mathrm{s}$ par son effet sur les trajectoires conventionnelles moyennes de grains de sable.

W étant la vitesse de chute en eau calme d'un grain, la trajectoire d'un grain de $1 \mathrm{~mm}$ est, sauf dans les couches du fond, peu différente de la résultante de $W$ et de U. Par contre, la longueur de la trajectoire d'un grain de $0,5 \mathrm{~mm}$ est celle de la résultante $\times 1,3$ et, pour un grain de 0,2 a $0,3 \mathrm{~mm}$, de $W=0,02 \mathrm{~m} / \mathrm{s}$ environ, le facteur 1,3 passe à 2. L'effet de la turbulence isotrope pent

(1) Voir "Description of the turbulence-meter "Saturnus" 》 (Hydraulic Labotatory Delft) donc être comparé à celủ d'une composante verticale de la vitesse qui serait $1 \%$ de la vitesse horizontale.

M. Montags ajoute que des cannelures longitudinales, dont l'effet est de réduire les mouvements tourbillonnaires partant du radier, réduisent la turbulence isotrope de près de $40 \%$ el qu'un tapis sans fin disposé sur le radicr et se déplaçant à la vitesse $U$ réduit les effets do la turbulence, donc, peut-on dire, la turbulence elle-mème, à zéro.

M. le Président conciut que la définition de la turbulence varie très sensiblement suivant les expérimentateurs qui domnent ce nom à des phénomènes très différents.

$2^{\circ}$ L'étude des conditions de début de mouvement des grains de matérian constituant le fond révèle que raction de la houle peut être notable, même par des fonds de plusieurs dizaines de mètres. Une houle d'amplitude totale $6 \mathrm{~m}$, longueur d'onde totale $120 \mathrm{~m}$, serait capable de mettre en mouvement un grain de sable de $0,3 \mathrm{~mm}$ par $60 \mathrm{~m}$ de fond.

M. le Président se demande si on peut extrapoler les résultats obtenus sur des houles de laboratoire par MN. VINCENT et RUELLAN à des houles de la nature qui mesurent $6 \mathrm{~m}$ d'amplitude totale, et se prodnisent $\dot{a} 60 \mathrm{~m}$ de profondeur.

M. De Rouvicte rappelle que, pour expliquer la quantité de sable qui est entrée dans l'estuaire de la Seine, il faut supposer qu'en plus de la destruction des falaises du Calvados, le fond, qui est à $16 \mathrm{~m}$, est remué par des houles de 4 ou $5 \mathrm{~m}$.

M. VINCENT pense que cette hypothèse est très probable, à condition que le fond soit constitué de grains suffisamment fins.

M. Vatemiors estime que l'on peut extrapoler le mou vement an voisinage du fond, du modèle réduit à la nature, puisque l'on peut caleuler ce monvement suivant une théorie vérifiée en première approximation et que l'on peut done le réaliser sur modèle réduit.

M. Vincent est, toutefois, bien d'accord sur le fait que sal conclusion repose sur une hypothèse explicitée dans le mémoire : à savoir que le fond est constitué de matém riaux bien nets; alors qu'en fait, souvent ces matériaux sont couverts de particules fines, voire de végétation.

Tout en soulignant l'intérêt des résultats de M. Vincent, qui constituent un progrès dans la sécurité de l'extrapoJation, M. LheruitTe rapporte la pensée de M. LARRAS, dont il déplore l'absence aujourd'hui, et se rapproche de cette pensée : le mouvement de grains isolés dépend essentiellement de la compacité du massif et des conditions très variables, et peut difficilement donner lieu à des lois. Il semble que le début de formations des rides puisse donner lieu à des lois probablement plus précises et que l'on puisse mienx traduire par des formules.

M. Lhermitre a comparé les différents critères proposès à la suite d'expériences faites en Amérique par Bagnold et Taylor, en France par MM. Fauquet et MartinotLagarde, à l'Institut de Mécanique des Fluides de Lille, par M. Larras (IV's Journées de l'Hydraulique et Annales des Ponts et Chaussées), par MM. VINGENT et Ruellay (SO.GR.E.A.H.), et par lui-même (L.C.H.F.). Ces formules font intervenir, en général trois paramètres : d'une part, l'amplitude $2 h$ et la période $2 \mathrm{~T}$ de la houle et. d'autro part, le diamètre des grains, $D$ (ou leur vitesse de chute).

a) l'exposant négatif $x$ du groupement : $h \mathrm{~T}^{--x}$ prend les valeurs suivantes, selon les auteurs :

M. Taylor $\ldots \ldots \ldots \ldots \ldots \ldots \ldots \ldots \ldots \ldots, x=3 / 2$.

MM. Fauquet of Martinot-Lagarde .... $x=4 / 3$.

M. Madhay Manohar . . . . . . . . . $x=1$.

M. Larras $\ldots \ldots \ldots \ldots \ldots \ldots \ldots \ldots \ldots \ldots, x \neq 1 / 2$. 
MM. Vincent et Ruellan .........., $1 / 2<x<1$. suivant prédominance des $U$ ou des $\bar{U}$ de leur formule.

M. Lhermitte . . . . . . . . . . . . . $x=1 / 4$ mais en faisant en définitive intervenir l'expression $2 h$ th $a \mathrm{H}$.

Seules, les deux premières de ces valeurs sont très supérieures à 1, probablement parce qu'elles correspondent $\grave{a}$ des houles ayant des harmoniques importants, alors que les autres formules correspondent à des houles relativement très pures.

b) L'exposant négatif $y \mathrm{du}$ groupement : $\mathrm{D}(2 h)^{-y}$ prend les valeurs suivantes, toutes de l'ordre de 2 , sauf pour M. Madhav Manohar :

MM. Bagnold et Taylor ............ $2<y<2,25$.

M. Martinot-Lagarde $\ldots \ldots \ldots \ldots \ldots \ldots, 2<y<2,25$.

M. Madhav Manohar ............. $y=5$.

M. Lhermitte ................. $y=2$.

M. Larras . ................. $1<y<2$. suivant les dimensions des grains.

MM. Vincent et Ruellan ............ $1<y<2$.

Les résultats des expérimentateurs ayant utilisé une houle réelle de laboratoire sont concordants entre eux. Ceux de Madhav Manohar, sont discordants, et mettent en évidence l'importance des forces d'inertie dans le processus utilisé par cet auteur.

En ce qui concerne le critère du début du phénomène, M. Lhermitre ajoute que MM. Vincent et Ruellan et M. Taylor prennent le début de mouvement des grains, tandis que M. Bagnold, MM. Martinot-Lagarde et Faucuet, M. Larras et M. Lhermitte prennent le début de formation des rides.

M. Vincent précise qu'il a fait en même temps l'étude du début de mouvement des grains et l'étude du transport de ces grains, si bien qu'il est arrivé à tracer une courbo du débit solide des grains en fonction des caractéristiques de la houle; il avait donc un point de débit nul qui précisait ce critère de début de mouvement.

$3^{\circ}$ L'étude expérimentale, comme la théorie en fluide visqueux, fait apparatre l'existence, au voisinage immédiat du fond, d'un courant d'entraînement des particules liquides portant tonjonrs dans le sens de propagation de la houle.

M. Vincent précise que la comparaison entre les valeurs du courant d'entraînement tel qu'il résulte de la théorie en fluides visqueux et les résultats avec les expériences d'écoulement de ces fluides au voisinage du fond dans un canal expérimental, est satisfaisante.

M. Lhenmitre confirme que d'après ses propres expériences, il existe, dans la couche limite d'un fluide (dans laquelle les phènomènes de viscosité sont prépondérants) un déplacement moyen dans le sens de progression do la hovle.

M. Vacenbors remarque que, selon des expériences faites en Angleterre, et qui seront publiées dans le compte rendu du Coastal Engineering, cette théorie n'est pas valable pour les fonds très petits. M. LHenmirte ajoute que le transport dans le sens de propagation de la houle cesse lorsqu'il y a une turbulence très forte sur le fond. M. Vincent estime que cela est bien difficile à observer dans un canal.

M. Lhermtre indique que les observations du Laboratoire Central d'Hydraulique de France confirment celles de MM. Vincent et Ruellan, savoir la présence de courants de masse dirigés lans le sens de propagation de la houle près du fond; courants observés pour la première fois en mer par M. Bertrand de Caligny.

M. Lhenarte rapporte une autre nouvelle remarque de $M$. Larras sur ces courants : ils sont déterminés mathématiquement d'une façon uniforme, à partir de la distribution du rotationnel dans le mouvement de la houle (travaux de Dubreil-Jacotin, Biesel et Kravtchenko). Pour déterminer ces courants ou la distribution du rotationnel, il faut rattacher ceux-ci aux conditions aux limites (extrémités du canal, en surface ou au fond) on aux conditions de propagation du mouvement dans la masse du fluide. En particulier en ce qui concerne les houles établies monochromatiques et périodiques, de laboratoire, la détermination de cette distribution près du fond est liée directement aux propriétés de la couche limite.

M. LHERMTte rappelle qu'il a indiqué, en avril dernier, dans une communication à l'Académie des Sciences, que la propagation d'un mouvement périodique dans le temps et dans l'espace, exige le déplacement de la couche limite de celui-ci dans le sens de la propagation de la houle, et, corrélativement, par suite du frottement, la génération d'une distribution de rotationnel décroissant depuis le fond jusqu'à la surface symétrique de la distribution du rotationnel décroissant de la surface au fond, que l'on connaît dans la génération de la houle par le vent. Ceci permet de généraliser la notion de houle normale, telle que M. Miche l'a exposée dans son livre sur Ies houles, et de compléter les équations du second ordre que cet auteur avait proposées (1).

M. DE Rouvilte demande si cela résoud le problème de l'existence d'un «courant de houle» qui s'est révélée dans la nature (entraînement des bateaux non amarrés, mouvements de sable) et par quelques expériences ele laboratoire, et semble s'expliquer par des effets de surface limite.

M. Vincent répond que la formation des plages avec une certaine pente paraît confirmer l'existence du courant de houle.

$4^{\circ}$ Ce courant d'entrainement (qui, en canal d'essais, s'accompagne d'un courant de transport dans lit masse fluide dont la distribution verticale varie selon les caractéristiques relatives all fuide) comme lu prépondérance, all voisinage du fond notamment, de la vitesse des particules liquides dans le sens de sa propagation donnent lieu généralement sur un fond horizontal à un «transport solide effectif» dans le sens de propagation de la houle.

M. Vincent indique que le transport solide effectif mentionné au paragraphe 4 de ses conclusions existe bien dans le eanal expérimental, et probablement dans la nature.

5" In fait de l'existence du contant de transporl de masse et de la mise en suspension des matériaut an-dessus du fond, un certain transport solide pent exister vers le large. Ces résultats permeltent d'expliquer qu'en houle allongée et régntière, les matériaux sont de préférence charriés dans le sens de la houle et viennent engraisser les rivages, tandis qu'en tempête une turbulence résultante inportante donne de la suspension et favorise l'érosion de ces rivages.

(1) Un travail de $M$. Lhermitte paraitra prochainement ì ce sujet. 
M. VINCENT indique que c'est tune conclusion valable en canal d'essai et fait un dessin au tableau pour l'expliquer.

M. Lhermerte a bien constaté, sur le fond d'un canal de laboratoire, la tendance à un courant d'entraînement vers le large; mais sa vitesse est moins forte que celui qui subsiste dans le sens de propagation de la houle, et comme, d'autre part, la distribution des matériaux va en décroissant, il est absolument improbable que le transport qui peut être emmené vers le large soit supérieur à celui qui se propage vers la côte.

$6^{\circ}$ Sur un fond en pente, le transport vers la cote est contrebalancé par l'effet de la gravité, les conrants provoqués par les vents di large et les conrants de densité prenant naissance dans la zone de deferlement si bien que finalement les matériaux érodés du continent se perlent, en partie, lentement, vers le large.

M. VINCENT indique que cette conclusion est tirée de l'action de la houle sur le fond en laboratore.

Comme conclusion générale, $M$. le Président estime qu'il est assez imprudent de tirer d'une seule expérience des gẻnéralisations trop étendues et, en particulier, d'extrapoler les rẻsultats d'études en laboratoire à ce qui peut se passer à la mer, où il n'y a pas de houles régulières ni de fonds réguliers.

M. de Rouvilie pense qu'il serait déjà très important de rapprocher les différentes définitions de la turbulence.

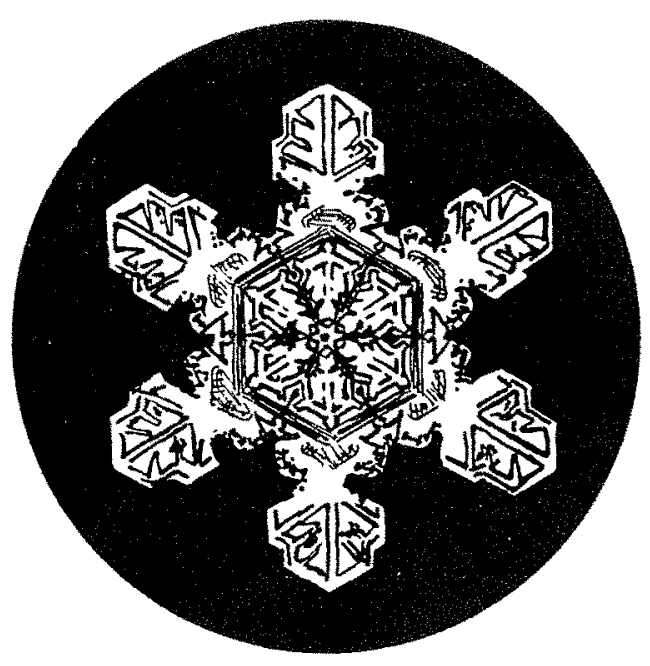

